has no cough, and her general state of health appears to be very good. On closely examining into the beat of the pulse at the wrist, I cannot detect that it is either small or weak; and the impression it gave to me was, that there seemed no blood in it. Such an affection of the heart as this is very rare, and I only remember to have seen one or two cases of it; yet, very strange to say, there is another case just like this in a boy in one of the wards upstairs. In each of these cases the symptoms are precisely alike, and there is no proportionate power whatever between the impulsive action of the heart in the chest and the beat of the artery at the wrist. In the former cases of a similar nature which $I$ have seen, I was induced to consider this affection as arising from over exertion; and in these cases now in the house, the same cause appears to have been in operation in producing the affection. The girl, of whose case I first spoke, has been a mursery servant, and has been, I suppose, accustomed to carry about a heavy child, and the heart has been thus, I may say, over strained. The boy has been working on board of some of the colliers, and has lifted heavy sacks of coals, and the same over straining of the heart has resulted. In both of these cases the heart appears too weak to empty itself of the blood which its cavities contain; and such may, doubtless, be the case when we refer to the very feeble arterial impulse at the wrist. Believing that there might be some inflammation of the internal structures of the heart, I ordered the woman to have a blister placed over the sternum, and to take some calomel and opium; and, under this simple treatment, the pulse at the wrist has much improved in tone and volume. I shall, I dare say, have occasion to draw your attention to these cases at a future time; they are very interesting, and well deserving of your attention.

\section{INFANTILE REMITTENT FEVER.}

This was formerly known by the title of worm fever, but this term has been nearly exploded from the scientific phraseology of medicine, since the peculiar inquiries which French physicians have made into the affections of the mucous membrane of the ali. mentary canal. This disease has been supposed to consist of a subacute inflammation of the bowels, but it has no relation whatever to this peculiar gastro. enteric affection. This infantile disease, denominated more properly remittent fever, is very commonly met with amongst the upper and lower classes of society, and arises in each of these from very opposite causes, but each tending to the same results. In the upper classes the children, when brought home from school at Christmas, are generally allowed their own way with respect to the quantity of food they shall eat; they make a hearty breakfast in the morning, and dine about the middle of the day, having frequently another dinner at a later period; to this succeeds tea, and perhaps in some cases supper.

Now, with all this quantity of food, and owing to the state of the weather, no opportunity of taking any out-door exercise or inhaling any fresh air, it is not to be wondered at that the bowels become loaded with unhealthy secretions, and fever is set up as the result of this. In the lower classes, where air and exercise are most abundant, but where food is for the most part of of a poor, scanty, and unwholesome nature, the children are fortunate if they get any food at all; they are content to have what is termed the " run of the house;" and if their parents can get bacon and potatoes, they get the same. But, after a time, the want of an adequate nutrition displays itself in a loaded and confined state of the bowels; there is a total loss of appetite, with great sluggishness and fever; the sleep is heavy, restless, and disturbed; the urine is scanty in quantity, and of a yellow colour, and looks like thick orange juice. Well, perhaps, when you are called in, you detect all these symptoms as being present in a greater or less degree, and you are told that the child has had some physic, and that the bowels are open. On examining the alvine secretions, you find them, perhaps, of a dirty watery character. Now, if you meet with such a state of things as this in a child of seven or eight years of age, you will find it necessary to give several large doses of calomel before the bowels will show any disposition to unload themselves properly, and this you must follow up with active purgation for some time before the child will be much improved. Now, this disease arises either from bad food in itsclf, or from an over-supply of good food, whereby the bowels become loaded, and vitiated secretions line the whole of the alimentary canal, and become absorbed into the general mass of circulating fluids. Dr. Butter wrote on this disease, and it was from him that it received the name of worm fever, and in his work he promulgated the fanciful and absurd theory that worms were sent to suck up the depraved and faulty secretions arising in this discase, and that they were, therefore, to be looked upon as a benefit sent by Providence to mankind, and not as an evil. But suppose a state of things such as $I$ have described to you be allowed to go on unchecked, you will find that these foul secretions which line the alimentary canal will in time become absorbed into the system the little patient gradually gets worse; he takes no food, and becomes weaker every day ; sleep and delirium will now be found to coexist together; and a severe febrile paroxysm ushers in the scanty and disturbed repose of each night; the abdomen becomes hard and swollen; the urine scanty and high-coloured; and eventually the early symptoms of effusion into the brain manifest themselves.

In treating this disease, the old physicians used to purge their patients to a very great extent; but these strong medicines will not bring away much feculent matter till after some considerable time has elapsed. The recovery of such cases is always long and tedious. I have seen many little patients whose bowels required to be continually stimulated, the intestines being filled with foul air and fouler secretions. I have been obliged to give repeated strong doses of calomel, followed up by brisk purgatives, and these means should be combined with a diet carefully regulated. In the case which we have had upstairs I gave rhubarb and calomel, and when the bowels had been well freed I gave canella, cascarilla, and soda, with animal broths and milk for diet, and such things as could be healthily absorbed, and form healthy secretions. There has been another case of remittent fever in the house, in which, from a predominance of acidity in the bowels, thore was frequent alvine evacuations of a foul and unwholesome character. This was treated with the hydrargyrum cum creta, and the pulv. ipecac. comp. with castor oil and rhubarb, to remove the diseased secretions, and beef-tea for diet.

\section{FIVE CASES}

OF

\section{STONE I N THE BLA D DER TREATED}

\section{BY LITHOTRITY.}

By T. P. Teale,

Surgeon to the Leeds General Infirmary.

Much uncertainty prevails in the minds of surgeons respecting the therapeutic value of lithotrity. 'That this operation has, in many instances, been advantageously employed, there can be no doubt; and it is equally true that it has sometimes greatly aggravated the sufferings of the patient. From the extreme paucity of recorded cases of lithotrity, since the operation by drilling was superseded by the more simple process now adopted, it is, perhaps, impossible at pre- 
sent to lay down positive rules for guiding the practice of the surgeon as to the adoption or rejection of lithotrity in any particular case; but our uncertainty in this respect might soon be remedied, if those surgeons who have practised lithotrity would place on record all their cases.

In order to supply a small share of information on this subject, I propose to relate five cases of stone in the bladder which have been treated by lithotrity. My object will be simply to relate the facts, without attempting to generalise from too limited a number of observations. It must, however, be stated that, after the painful experience furnished by the fifth case, I should be unwilling again to adopt lithotrity when the stone is of so large a size, however readily it might admit of being seized, and however free from morbid irritability the bladder might be.

When the drilling and percussing processes were adopted for the disintegration of the stone, I did not consider that the operation had attained that degree of simplicity which would justify its general adoption; and it was not until the introduction of Mr. Weiss's screw-lithotrite that I determined upon practising lithotrity in such cases of stone in the bladder as should appear favourable for this operation. Since that time I have had occasion to operate twelve times for vesical calculus. On seven of these occasions lithotrity was deemed inadmissible; in five, from sinall size of the urethra, the subjects being children; in one, from chronic stricture and irritable bladder and in another, from large size of the stone. These cases were treated by lithotomy. Six of the operations were successful; the seventh proved fatal on the twenty-first day. The subject of this case was a man aged 40 , from whom a lithic acid calculus, weighing nearly three ounces, was extracted without difficulty, or any unfavourable occurrence, in two minutes and a half. He appeared perfectly well for a few days, after which he was seized with pneumonia; the wound became sloughy; erysipelatous inflammation spread over the perineum and nates, which became extensively excoriated. Under these affections he gradually sunk. A post-mortem examination was not allowed.

In these operations the straight staff and knife of Mr. Key were employed; and it affords me great pleasure to bear testimony to the value of this gentleman's proposal of effecting the section of the prostate on a straight director, whereby the difficulty and danger of lithotomy are greatly mitigated. The section of the prostate in all the cases was of very limited extent, being merely sufficient to allow the introduction of the forefinger, the opening being afterwards dilated by the finger in children, and by the blunt gorget in adults. A gum-elastic tube was introduced into the bladder, and allowed to remain for a period varying from eight to forty-eight hours.

Of the five cases treated by lithotrity, four were perfectly successful. One of these patients, after enjoying ease and health for upwards of a year, again felt symptoms of stone, when a small concretion was detected by the sound, and was removed by lithotrity at two sittings. The fifth case was for a short time apparently cured; but the symptoms of stone soon recurred in a most aggravated form, and lithotomy was performed under very unfavourable circumstances.

CaSE I.-Lithotrity-Five Sittings-Cure.-John Ruttar, of Howgill, near Gisburn, husbandman, aged 56 , of temperate habits and healthy aspect, was admitted into the Leeds Infirmary, November 16, 1836, on account of stone in the bladder.

He has always enjoyed good health, with the exception of his urinary complaints. About nine years ago he first felt some uneasiness in voiding the urine, which was high coloured, and deposited a red sediment. This condition of the urine continued until within a recent period. About six weeks ago he experienced a severe attack of pain in one of the kidneys, extending down the ureter, which was followed by pain in the bladdex, especially after micturition, and occasionally by the appearance of blood in the urine. When admitted in to the hospital, a stone of moderate size was detected by sounding. The prostate was healthy, and the bladder not particularly irritable.

Nov. 22. The patient being placed on Heurteloup's bed, and the bladder injected with eight ounces of tepid water, a stone, measuring five and a half lines, was readily seized, and crushed once by Weiss's screw-lithotrite. Very slight pain was experienced during the operation, after which the patient was sent to bed, and placed on low diet.

23. He had a slight rigor during the night, which was followed by thirst, and some heat of the skin; pulse, 100 ; tenderness on pressing the pubic region. Several small fragments of calculus have been voided along with the urine. Six leeches to be applied above the pubes, and frequent hot fomentations.

24. Pulse, 90, soft; skin cool; pain relieved.

29. Portions of stone have been discharged daily since the operation.

Dec. 10. Lithotrity repeated; the stone was seized and crushed three or four times.

20. Lithotrity repeated.

29. Lithotrity repeated, with the screw-scoop, which was introduced three times, and withdrawn each time loaded with calculous matter.

31. The screw-scoop was again introduced twice, and several small fragments were crushed and extracted.

Jan. 10. After each of the four last operations, fragments of stone were discharged with the urine for a few days. They were soarcely attended with any pain, and were not followed by any symptoms of irritation. During the last four days he has been free from all symptoms of stone, and, on sounding, no portions of calculus can be detected in the bladder.

He left the hospital a few days afterwards in perfect health.

In a letter dated January 4, 1841, he states that he has enjoyed excellent health since leaving the infirmary, and that he had been able uninterruptedly to attend to his laborious occupation.

According to the analysis of $\mathrm{Mr}$. West, lecturer on chemistry in the Leeds School of Medicine, some of the fragments consisted of lithic acid, and others of the mixed phosphates.

CASE II. - Lithotrity - Three Sittings-Cure.George Rushforth, of Bughouse, aged 30, was admitted into the infirmary May 23, 1839, with stone in the bladder. The urethra was capacious; bladder and prostate healthy. No symptoms of irritation followed the operation of sounding, and the stone was evidently of small size.

June 1. The stone was readily seized and crushed by the screw-lithotrite. The pain attending the operation was very slight, but it was followed by feverishness, and inflammation in one testicle, on which account the repetition of the operation was postponed several days. Numerous small fragments were discharged for a few days after the operation.

Lithotrity was repeated with the screw-scoop on the 18th and 22nd of July, without any unpleasant consequences.

July 26. Since the last operation he has been free from symptoms of stone, and no calculus can now be detected on sounding.

He left the hospital a few days afterwards perfectly relieved.

In a letter dated December 28, 1840, he states " that he is in perfect health, and has not had any retum of the symptoms of his late troublesome malady."

The stone consisted of lithic acid, with slight inter- 
mixture of phosphate anc carbonate of lime (Mr. West).

CASE III.-Lithotrit?-Female-Three SittingsCure.-Oct. 29, 1839. Hannah Squires, of Bramley, near Leeds, was adnitted into the infirmary with stone in the bladdes. On her admission the bladder was extremely irrituble, and the urine deposited an abundance of mucc-purulent matter. On the 12th of December, the morbid condition of the bladder was so far relieved as to allow of lithotrity being performed, which was repeated on the 2lst of December and on the 7 th of Jamiary, after which she returned home quite well. In January, 1841, she was in good health, and had not experienced any return of the complaint.

This case is recorded more fully in the "Provincial Medical and Surgical Journal," for December, 1840.

Case IV.-Lithotrity-Six Sittings-Cure.-After Tuelve Months a Second Occurrence of Stone-Lithotrity-Tuo Sittings-Cure.-William Harwood, of Holbeck, aged 63 , was admitted into the infirmary, Oct. 9, 1840, having suffercd from symptoms of stone in the bladder about three years. His general health was good, and his bladder and prostate healthy.

Lithotrity was performed on the 12th of October. The stone measured seven lines. He suffered very little pain from the operation, but on the 16th the urine deposited a considerable quantity of viscid transparent mucus. Several portions of calculus had been discharged. Under the use of oil of cubebs the mucus gradially disappeared.

The operation was repeated on the 7 th and 25 th of November, and on the 5th, 19th, and 26th of December; but in none of these instances was it followed by catarrh of the bladder, nor any other inconvenience. The symptoms being now relieved, and the sound failing to detect the presence of the stone, he left the lospital in perfect health.

Some of the fragments of stone consisted of lithic acid, and others of phosphate of lime.

After remaining perfectly well upwards of twelve months, he again experienced pain after passing the urine, and was re-admitted into the infirmary, when a small stone was detected by sounding. On the 24 th of February, 1842, the screw-scoop was introduced and a stone, measuring three lines, was seized and crushed. Between the blades of the scoop was brought away a fragment of stone, which was cvidently the rounded extremity of the concretion, and consisted of very compact lithic acid. On the 28 th, three or four fragments, the largest of which measured five lines, were crushed. During the two following days numerous comminuted portions were discharged. He did not suffer any inconvenience from either of these operations, but continued to walk about the wards as usual. On the 3rd of March he stated that he was quite free from the symptoms of stone. He was placed on Heurteloup's bed, and the bladder, being injected, was sounded by the three surgeons of the infirmary, but no fragment of stone could be detected. The patient, however, was ordered to remain in the hospital a few days longer, that the bladder might be again examined.

CASE V.-Lithotrity-Eight Sittings-Apparent Cure-Speedy Relapse-Lithotomy.-Nov. 27, 1840. In conjunction with Mr. Bailey I visited Mr. J. M., of Upleatham, in the North Riding of Yorkshire, aged 72, a fine, healthy-looking man, of tranquil and somewhat phlegmatic temperament, who had been afflicted with stone in the bladder about three years. Of late his sufferings have been extreme; the slightest exereise in walking or riding is followed by a bloody state of the urine, and he is quite unable to bear the motion of a carriage. He has hitherto borne the operation of sounding without inconvenience. The prostate is somewhat enlarged, but not tender on pressure; the the urethra capacious. Being unable to perform a journey of seventy miles to Leeds, he was particularly urgent that some means should be attempted for his relief at his own home.

Having injected the bladder with six ounces of tepid water, which it readily received, I introduced the lithotrite; and found that, although the prostate was large, it did not constitute any impediment to the seizure of the stone, which measured an inch and a half by the scale. The screw was then put in action, and the stone crushed. This process was repeated four or five times. He suffered so little that he thought it was only the sounding that had been performed, and that some process more painful was to follow.

By a letter from Mr. Bailey a few days afterwards, I was informed that our patient passed a good night after the operation, but that on the following day he had a smart rigor, and felt some uneasiness from the passage of fragments of stone. An opiate procured him an exccllent night, and on the next day he was comfortable and free from fever; pulse 75 ; tongue moist and clean. He continued to pass numerous fragments of stone for several days. The urine was clear and frce from mucus.

Dec. 15. I again visitcd him, and found that during the last four or five days he had felt some pain in the bladder, accompanied by a severe sense of bearing down at the rectum. The urine had deposited about two ounces of viscid mucus daily. Tongue clean and moist; pulse 75 . On attempting to inject the bladder, the passage of the fluid was resisted much more than on the former occasion, and the distension of the bladder was attended with more pain. The passage of the catheter through the prostate gave considerable uneasiness. On examination by the rectum the prostate was found to be tender on pressure. Under these circumstances the operation of lithotrity was postponed, and the following treatment prescribed :-

Six leeches to the anus every second day ; fomentations to the pubic and perineal regions morning and evening. A draught, containing oil of cubebs and liquor potassa, to be taken three times daily ; diluents.

Dec. 31. Finding him perfectly relieved by this treatment, I repeated lithotrity with very little pain or inconvenience. A large quantity of detritus followed this operation, and in a few days the relief of his old symptoms was so great that he walked about his farm with considerable comfort, and could take moderate exercise without the urine becoming bloody.

On the 14th of Jamuary he felt so much easier that he undertook the journey to Leeds by chaise and rail. way, and performed it without any material inconvenience, and without the urine exhibiting any appearance of blood.

Lithotrity was repeated on the 19th and 26th of January, and on the 2nd and 7 th of February.

On the 11th of February he was scized with influenza, then epidemic, on which account the operation was postponed until the 20th, and was repeated on the 26th.

Two days after the last operation he felt quite free from the symptoms of stone, and at several subsequent soundings none could be detected. Being extremely anxious to return home, he left Leeds on the 5th of March, feeling perfectly well.

The fragments of stone in this case were of the colour of box-wood, and consisted of lithic acid with a slight intermixture of the phosphates.

His journey was performed with perfect comfort, and for about a week he took exercise freely on horseback, and was greatly delighted with the relief he had obtained. Unfortunately, lowever, the respite was but of short duration. At the middle of March he began to have pain in the bladder at nights, was obliged to rise frequently to pass urine, and felt pain at the end of the penis after micturition. The urine also deposited mucus.

On the 15th of April he returned to Leeds. The bladder at that time was extremely irritable; the urine deposited abundance of muco-purulent matter, 
was loaded with the phosphates, and occasionally tinged with blood. The presence of calculi was readily detected by the sound. The bladder would scarcely bear the injection of half an ounce of fluid.

Leeches were applied to the perineum at intervals of two or three days. The bladder was injected daily with tepid water containing morphia; and he took the decoction of Pareira.

May 13. The irritability of the bladder being considerably diminished, Weiss's screw-scoop was introduced; portions of calculus were seized and crushed three or four times, and the instrument was withdrawn loaded with soft fragments of a white colour, consisting of the mixed phosphates. During several following days, portions of calculous matter were discharged.

The operation was repeated on the 31st of May and on the 7 th and 25 th of January. On the two latter occasions, I had the co-operation of my colleague, Mr. Smith. During the intervals of these operations, leeches, sedatives, and diluents were employed. Considerable quantities of the white soft calculous matter were discharged. The bladder, however, became more irritable, and it was deemed inadvisable to persevere in any fiurther at tempts at lithotrity.

He returned home much dispirited and in a state of great suffering, and on the 8th of August I went over to his residence to attempt his relief by lithotomy.

Assisted by Mr. Bailey, Mr. Bird, and Mr. Dowell, I performed the lateral operation on the 9 th of August, and speedily brought away, in the first grasp of the forceps, three calculi, about the size of hazel nuts, which, on subsequent examination, were found to consist of the mixed phosphates, with a small nucleus of lithic acid, nearly pure. A fourth stone of the same character, and numerous small soft concretions of the phosphates, resembling mortar, were removed by the scoop. The bladder was freely injected with tepid water, which brought away an abundance of gritty particles. Similar particles were felt, on the introduction of the finger, adhering to the mucous membrane of the bladder, and considerable time was occupied in their removal. When no more gritty matter came away with the injection, or could be felt by the finger or the sound, a gum -elastic tube was introduced, and the patient removed to bed.

He suffered but little constitutional irritation from the operation; the wound slowly healed; the urine soon began to deposit mucous and a thick layer of the phosphates; cloths, which had been wetted with the urine, after becoming dry, were loaded with phosphates in a pulverulent state.

Nov. 15. Mr. Bailey informs me, by letter, that the phosphates have lately been discharged as distinct concretions. Some of these, which he sent me, vary in size from that of a pin's head to a grain of wheat.

It was now suggested that the bladder should be frequently injected with tepid water, slightly acidı!lated with nitro-muriatic acid.

Feb. 8, 1842. Mr. Bailey states that, for the first few weeks, during which the acid injection was em. ployed, the mucous secretion diminished, the urine improved in appearance, the pain abated, the earthy concretions were frequently discharged, and the general health was good; but that, during the last month, the symptoms have been aggrarated, no concretions have been discharged, and a small calculus can now be distinctly felt on introducing the catheter.

\section{ON TIIE}

\section{FIRST DENTITION OF INFANTS}

\section{By M. Trousseau,}

\section{Physician for Diseases of Women and Children} at the Necker Hospital.

In this memoir $M$. Trousseau gives an account of the phenomena of dentition in 6 infants. A much greater number had been examined, but the author prudently takes account only of those cases upon which he could place perfect reliance. The following are the principal results at which M. Trousseau has arrived; our readers may compare them with the results of Dr. Ashburner's experience, contained in an excellent little treatise "On Dentition and some Coincident Disorders :" -

\section{Period of Eruption of the First Tooth.}

The period at which the first tooth made its appearance was determined, without chance of error, in 25 infants-viz, 13 boys and 12 girls. For the males, two at 2 months; two at 4 ; two at 5 ; two at 6 ; two at 10 ; two at 11 ; one at 14 months. Extremes, 3 and 14 months; mean, 7 months.

For the females, one at 2 months; two at 5 ; one at 4 ; one at 5 ; three at 6 ; one at 7 ; two at 9 ; one at 14 months. Extremes, 2 to 14 months; mean, 6 months.

From these two tables, it appears that the period of eruption of the first tooth varies between 2 and 14 months, and that the mean period is $6 \frac{1}{2}$ months; hence, should these results be confirmed by further investigation, we shall have to modify the prevailing opinion, that the first tooth generally comes forth about the eight month.

The first milk tooth is generally the middle lower incisor; in 28 cases, this occurred 25 times; in two, the first tooth cut was a superior middle incisor; and in one, a first molar tooth.

\section{Eruption of the Second Tooth.}

Of the 25 infants just noticed, all cut an inferior middle incisor for the second tooth. Of the remaining three, ore cut two superior middle incisors; another, a lewer middle incisor after the superior incisor; the third cut four molar teeth in succession, before any of the middle teeth appeared. As a general rule, then, we may say that the second tooth which is cut is a middle lower incisor. The common opinion is, that the two first teeth appear nearly at the same time, and this appears to be true; thus, of 25 infants, the interval between the eruption of the first and second tooth was, in four cases, 1 day ; in two, 2 ; in one, 3 ; in three, 8 ; in seven, 15 ; in one, 30 ; and in one, 90 days. In six cases the author was unable to ascertain with certainty the period. Hence, the mean interval between the first two teeth is 13 days.

\section{Eruption of the other Incisors.}

After the inferior middle incisors came the superior middle. Of 18 infants, in whom the order of dentition was carefully observed for this purpose, 15 cut the teeth just mentioned, after their inferior middle incisors; one ahild had four lower incisors before a superior one appeared; another cut a superior lateral incisor before the middle one; and the third infant cut, alternately, an inferior middle incisor, then a superior one, then an inferior, and lastly a superior.

Generally speaking, then, the two upper middle incisors follow the two lower, but at a larger interval than is generally supposed. Thus, in 13 infants, this interval between the appearance of the lower and upper middle incisors was, in two, 8 days; three, 1 . month; three, 2 ; one, 3 ; one, 4 ; two, 5 ; and one, 10 months.

The differences here indicated are too great to admit of our determining with any accuracy the period at which the two superior middle incisors appear after the inferior ones. We have now the four lower first teeth, two below and two above; for the remainder, authors tell us that the two lower lateral incisors generally follow the middle superior ones, and are succeeded by the two superior lateral incisors. Of eleven children, examined to ascertain the point, nine had the superior lateral incisors before the lower ones; one had the lower lateral incisors before the middle 